\title{
Dynamic behaviour of core-shell structured Si nanoparticles during lithiation/delithiation cycling at dynamic loadings
}

\author{
Xiang Gao ${ }^{1,2}$, Chunhao Yuan ${ }^{1,2}$, and Jun $\mathrm{Xu}^{3,4^{*}}$ \\ ${ }^{1}$ Department of Automotive Engineering, School of Transportation Science and Engineering, Beihang University, Beijing, China \\ 2 Advanced Vehicle Research Center, Beihang University, Beijing, China \\ ${ }^{3}$ Department of Mechanical Engineering and Engineering Science, The University of North Carolina at Charlotte, Charlotte, NC \\ 28223, United States \\ ${ }^{4}$ Vehicle and Battery Safety Laboratory, The University of North Carolina at Charlotte, Charlotte, NC 28223, United States
}

\begin{abstract}
Dynamic behaviours of the core-shell structured (CSS) Si nanoparticles during electrochemical cycling were modelled. A core-shell-structured model that contained Si nanoparticles wrapped with amorphous carbon (a-C) was established. The system was charged and discharged under constant voltage with a dynamic loading applied on the surface of the particle. The volume change and associated stress simulated by the model without external loading were compared with the previous work done by other researchers and matched well. Different imposing moments of the external loading were introduced into this model to see the dynamic effects on the cycling behaviour of the Si particles. The present study illustrate the dynamic behaviour under external loading, which would give guidance for the Si based battery design and help to understand the coupling effects of dynamic loading and electrochemical cycling on Si anodes.
\end{abstract}

\section{Introduction}

Silicon is one kind of promising electrode materials for Lithium-ion batteries in the near future because of its high capacity $(3579 \mathrm{mAh} / \mathrm{g})[1,2]$. But the large volume expansion/contraction of Si during lithiation/delithiation cycling has been the main barrier of its broad application [3]. These volume changes would lead to significant contact stress between particles and then the stresses could cause fracture of particles or delamination of binder from particles. The fracture and delamination could lead to isolation of active particles then the electrical network was damaged. This is the main mechanism of the capacity fading in practical silicon based electrodes [4].

In order to reduce the impact of this volume change, lots of managements have been proposed in the $\mathrm{Si}$ electrode. The fracture can be mitigated by nanostructuring the silicon anodes [5-7] or compositing $[8,9]$ and so on. Core-shell structure is a typical configuration in stress managements of $\mathrm{Si}$ anode and some numerical studies have been carried out on this structure [5]. For example, Zhao et al. [10] studied the fracture and debonding in lithium-ion batteries with electrodes of hollow core-shell nanostructures. Liu et al. [11] built up an analytical model on stress-regulated lithiation kinetics and fracture of a similar yolk-shell structure.

Except for the capacity fading caused by the cycling degradation of the active Si particles, severe damages caused by abrupt dynamic loading ( which may occur in the driving of electric vehicle or accidentally falling of the mobile phone, and other common circumstances) is another main factor causing battery failures. Nonetheless, few works were done about the dynamic behaviours of CSS Si nanoparticles at high strain rates caused by external loadings. In this study, a simple finite element model was constructed to analyse the mechanical behaviour of two contact CSS particles with or without external dynamic loadings. The main purpose of this study is to understand the effects of dynamic loading on deformation behaviour and contact stress evolution during electrochemical cycling.

\section{Method}

Deformation and permanent shape changes in CSS nanoparticles and the evolution of the contact stress between two particles at external dynamic loading during electrochemical cycling were simulated using the software COMSOL. As shown in Fig. 1, two spherical Si particles with radius $\mathrm{R}=50 \mathrm{~nm}$ which were covered with a layer of amorphous carbon with thickness $t=10 \mathrm{~nm}$ were constructed in the simulation. The electrochemical and mechanical boundary conditions of the two particles were also shown in Fig.1. A constant current (or Li flux) were applied on the surface of the Si particle on the assumption that the diffusion of $\mathrm{Li}$ in amorphous carbon was ignored. The influence of the stress on diffusion of $\mathrm{Li}$ in $\mathrm{Si}$ particle were ignored in this study, so the diffusion of $\mathrm{Li}$ was assumed to be driven only by the gradient of concentration and was modelled with a constant $\mathrm{Li}$ diffusivity $\mathrm{D}=10^{-16} \mathrm{~m}^{2} / \mathrm{s} \quad$ [12]. For the

*Corresponding author: jun.xu@uncc.edu 
mechanical boundary conditions, symmetric boundary conditions were applied on the top and bottom surfaces of the two contact particles and the spherical surfaces of the particle were set to free boundary, shown in Fig. 1(a). When considering the external loading, the top particle was changed into a whole sphere and a plate was applied on the top of the particle with an initial speed $\mathrm{v}_{0}$ imposed at different moments during the electrochemical cycling, shown in Fig. 1 (b).

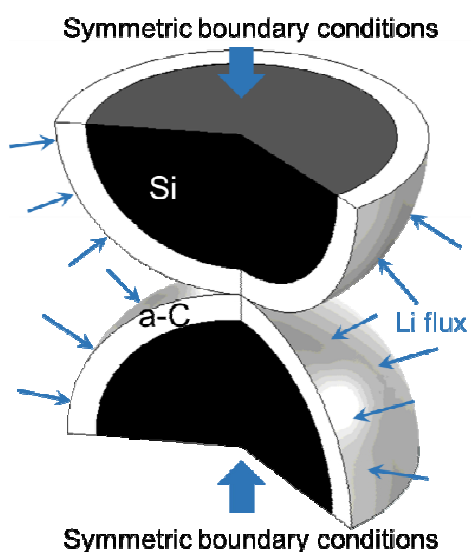

(a)

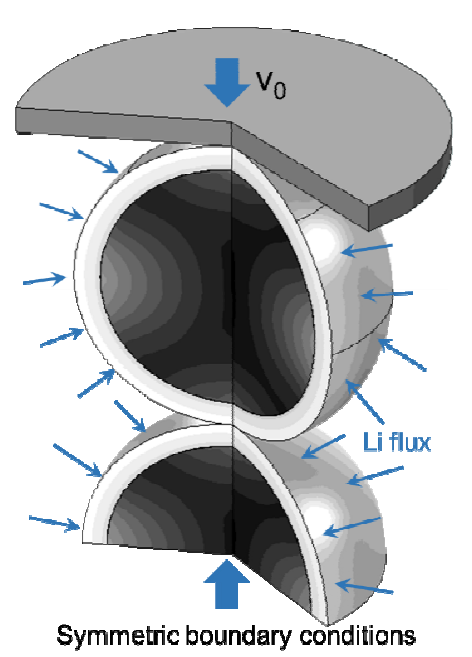

(b)

Fig. 1. Axisymmetric finite element model for core-shell structured Si nanoparticle. Black and gray represent Si particles and amorphous carbon, respectively. Thin blue arrows indicate $\mathrm{Li}$ flux and symmetric boundary condition were applied on bottom surfaces. A symmetric boundary condition was applied on top surface for (a) and a plate with a velocity imposed at different moment was applied on the top surface for (b).

The lithiation induced elastic-plastic deformation of Si particles is described by the elastic and perfectly plastic model [13]. The total true strain, $\varepsilon_{i j}$, can be written as

$$
\varepsilon_{i j}=\varepsilon_{i j}^{l}+\varepsilon_{i j}^{e}+\varepsilon_{i j}^{p}
$$

where $\varepsilon_{i j}^{l}, \quad \varepsilon_{i j}^{e}$ and $\varepsilon_{i j}^{p}$ represent the lithiation induced strain, elastic strain and plastic strain, respectively. The volume of the particle, $V$, is taken to be linear of the concentration of $\mathrm{Li}$,

$$
V=V_{0}(1+\alpha \bar{c})
$$

where $V_{0}$ is the initial volume of the particle, $\alpha$ is related to the volumes of atomic by $\alpha=2.7$ from the volume expansion $270 \%$ at full lithiation, i.e., $\mathrm{Li}_{15} \mathrm{Si}_{4}$ [14], and $\bar{c}$ is the normalized Li concentration defined as $\bar{c}=c / c_{\max }$. Then lithiation induced strain can be expressed as

$$
\varepsilon_{i j}^{l}=\ln (1+\alpha \bar{c}) / 3
$$

The elastic strain is given by Hook's law,

$$
\varepsilon_{i j}^{e}=\left[(1+v) \sigma_{i j}-v \sigma_{k k} \delta_{i j}\right] / E
$$

where Young's modulus, $E$, is $\mathrm{Li}$ concentrationdependent and can be written out based on firstprinciples [15] as

$$
E(\bar{c})=E_{0}+\bar{c}\left(E_{1}-E_{0}\right)
$$

where $E_{0}=112.4 \mathrm{GPa}$ corresponding to pure Si (i.e., $\bar{c}=0)$ and $E_{1}=50 \mathrm{GPa}$ corresponding to fully lithiated Si (i.e., $\bar{c}=1$ ). The plastic strain can be expressed as a generalized form,

$$
\varepsilon_{i j}^{p}=\lambda S_{i j}^{p}
$$

and is assumed to obey the classic $\mathrm{J}_{2}$-flow rule that the plastic yielding occurs when the von Mises stress, $\sigma_{e}=\sqrt{3 S_{i j} S_{i j} / 2}$, reaches the yield stress, $\sigma_{y} . S_{i j}$ is the deviatoric stress by $S_{i j}=\sigma_{i j}-\sigma_{k k} \delta_{i j} / 3$. The yield stress of the Si particle is also assumed to be dependent on the concentration [16],

$$
\sigma_{y}(\bar{c})=\sigma_{y}^{0}+\bar{c}\left(\sigma_{y}^{1}-\sigma_{y}^{0}\right)
$$

where the $\sigma_{y}^{1}=1.5 \mathrm{GPa}$ for $\bar{c}=0$, and $\sigma_{y}^{0}=0.6 \mathrm{GPa}$ for $\bar{c}=1$.

In this model, the amorphous carbon layer is assumed to be a simple linear-elastic material without $\mathrm{Li}$ diffusion, so the carbon layer only has mechanical deformation. The elastic modulus and Poisson ratio of the material are assumed to be $E_{c}=20 \mathrm{GPa}$ and $v_{c}=0.3$, respectively.

In the simulation process, a constant current or constant flux of Li was imposed on the surface of the $\mathrm{Si}$ particles. The charging/discharging rate in this study was $\mathrm{C}=0.25$. For the dynamic cases, six different moments at which a sudden velocity (strain rate: $\sim 100 \mathrm{~s}^{-1}$ ) was imposed on the top plate were selected to study the effects of dynamic loading on the deformation and stress evolution during charging and discharging cycling.

\section{Results and discussion}

\subsection{Model validation}

Before the dynamic behaviour was studied, electrochemical cycling induced deformation and stress evolution without external dynamic loading during two cycles was first investigated to make sure the accuracy of the model. Fig. 2(a) shows the evolution of contact 
stress between two $\mathrm{Si}$ particles without a-C covered. It is clear that the contact stress evolves nonlinearly in the first cycle while the stress evolution curve is almost linear in the subsequent cycle. It is indicated that the permanent shape change and most of the plastic deformation mainly occur in the first cycle. The nephogram in Fig 2(a) shows that the particles in the end of the first cycle $(\mathrm{t}=2.5 \mathrm{~h})$ produce permanent shape change while the shape remain almost no change in the end of the second cycle, which could also give support to this conclusion. As for the peak value of the contact stress, it is highest in the first cycle and decreases during the sequence cycles.

Then, a same case was simulated on two contacting CSS Si particles. The stress evolution and deformation of the particles are shown in Fig. 2(b). Compared to the stress evolution of the pure Si particles in Fig. 2(a), the peak value of the stress is smaller and the time window of one cycle is longer for the CSS particles. Note from the nephogram of point $b$ in Fig 2(b) that, there is almost no permanent shape change in Si particles in this case. The results show that the core-shell structure could help to reduce the contact stress of the Si particles and is an efficient strategy for stress management. The results of this model is consistant with the study done by other researchers [17]. So the model is validated and will be used in the studies of dynamic behaviours.

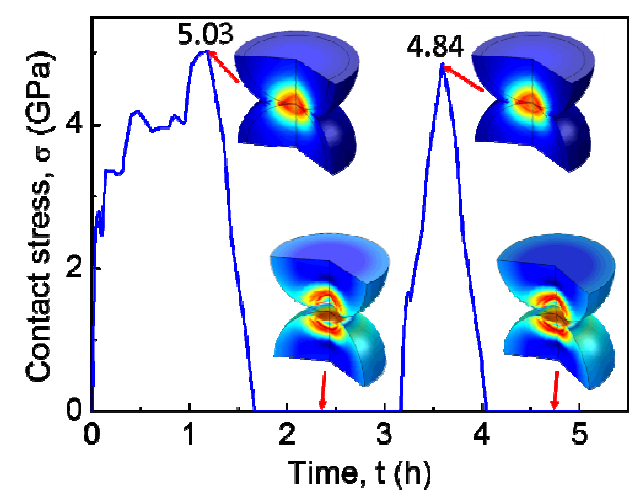

(a)

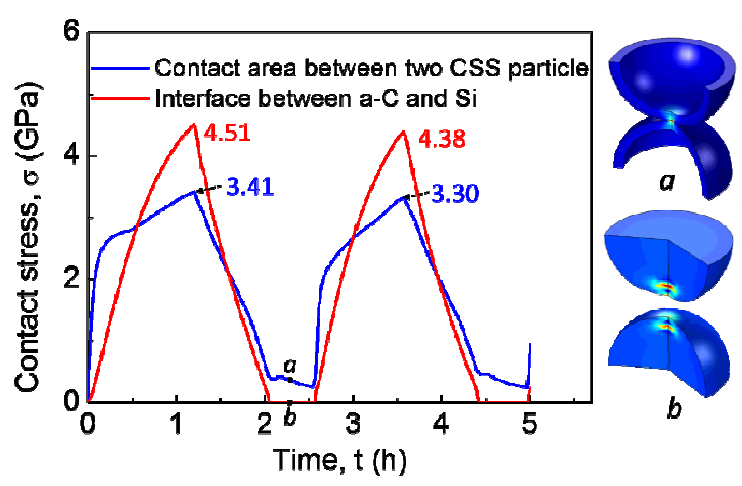

(b)

Fig. 2. Contact stress evolution and shape change during two electrochemical cycling without external dynamic loading of: (a) pure Si particle contacting pair and (b) CSS particle contact pair.

\subsection{Effects of external dynamic loading}

A plate with same velocity imposed at different moment during one electrochemical cycling was impact on the top particle, as shown in Fig. 2(b), in order to study the effects of external dynamic loadings on stress evolution. Six moments in one cycle were selected. Three of them $(2640 \mathrm{~s}, 3140 \mathrm{~s}, 3640 \mathrm{~s})$ were in lithiation process while the other three $(5000 \mathrm{~s}, 5500 \mathrm{~s}, 6000 \mathrm{~s})$ were in delithiation process. Fig. 3(a) and (b) show the stress evolution at the interface between $\mathrm{Si}$ particle and a-C layer and at the contact area between two CSS particle, respectively. It is indicated that a suddenly imposed velocity (or impact) would cause a sharp increase of contact stress. And the increase in the contact area between two CSS particles is larger than that at the interface between Si particle and a-C layer. So when the CSS particles based anode is impacted, the covering layer (a-C in this study) will be destroyed first and absorb a part of impact energy. This confirms one of the advantages of core-shell structure. It can be calculated from Fig 3(a) or (b) that same impact energy would cause almost same increment of the contact stress no matter in the lithiation or delithiation process. But the increase of the stress caused by impact in lithiation process is more likely to be kept, as shown in Fig 3(a). It may be attributed that the particles are still swelling after the impact which makes a tighter contact between particles. In contrast, the shrink of particles in the delithiation process will help to decrease the stress increment. Moreover, the impact in the lithiation process, especially near the end of the lithiation process, is more dangerous due to the larger peak value of the contact stress caused by it. The result showed in this work would give a simple understanding on the effects of external dynamic loading on the stress evolution in the particle scale.

\section{Conclusion}

Dynamic behaviours of the core-shell structured (CSS) $\mathrm{Si}$ nanoparticles during electrochemical cycling were modelled. A core-shell-structured model that contained Si nanoparticles wrapped with amorphous carbon was established. The system was charged and discharged under constant voltages with a dynamic loading applied on the surface of the particles. The model was validated by comparing the results without external loading with the previous work done by other researchers. The results of simulation on effects of the external loading draw the following conclusions: a) the stress increment caused by the dynamic loading in the contact area between two CSS particle are larger than that at the interface between $\mathrm{Si}$ particle and a-C layer; b) same impact energy would cause almost same increment of the contact stress no matter in the lithiation or delithiation process; c) the increase of the stress caused by impact in lithiation process is more likely to be kept and is more dangerous. The present study illustrates the dynamic behaviour under external loading, which would help to understand the coupling effects of dynamic loading and 
electrochemical cycling on $\mathrm{Si}$ anodes. More detailed works should be done to give more information for electrode design in the future.

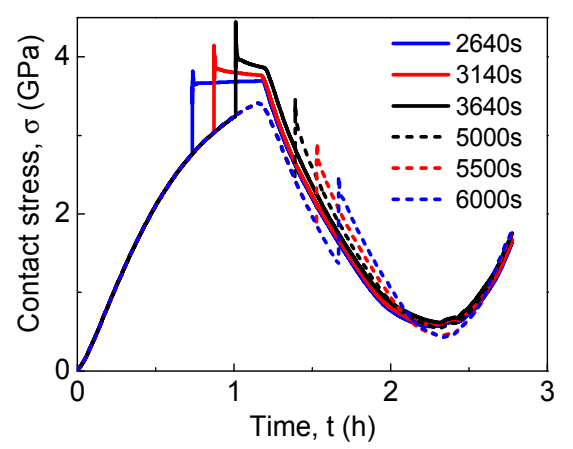

(a)

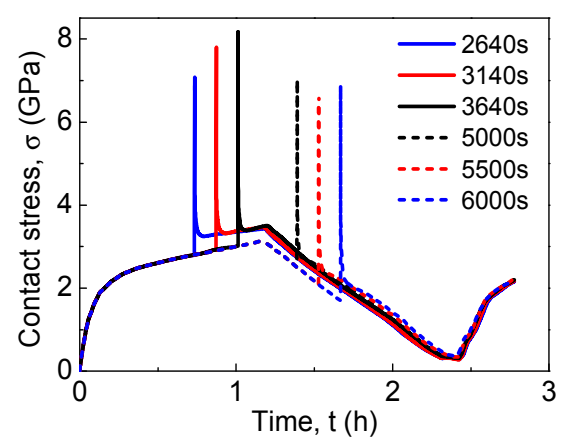

(b)

Fig. 3. Contact stress evolution of two contact CSS particles with external dynamic loading imposed at different moment in one cycle: (a) contact stress at the interface between Si particle and a-C layer; (b) contact stress in the contact area between two CSS particles.

This work is financially supported by Opening project of State Key Laboratory of Explosion Science and Technology (Beijing Institute of Technology) (KFJJ17-13M), Research Project of the State Key Laboratory of Vehicle NVH and Safety Technology under Grant NVHSKL-201610.

\section{References}

1. M.N. Obrovac, L. Christensen. Electrochem. Solid ST. 7, (2004)

2. U. Kasavajjula, C. Wang, A.J. Appleby. J. Power Sources. 163, 1003-39 (2007)

3. L.Y. Beaulieu, K.W. Eberman, R.L. Turner, L.J. Krause, J.R. Dahn. Electrochem. Solid ST. 4, (2001)

4. G. Liu, H. Zheng, A.S. Simens, A.M. Minor, X. Song, V.S. Battaglia. J. Electrochem. Soc. 154, 118 (2007)

5. X. Li, P. Meduri, X. Chen, W. Qi, M.H. Engelhard, W. Xu. J. Mater. Chem. 22, 11014-7 (2012)

6. R. Huang, X. Fan, W. Shen, J. Zhu. Appl. Phys. Lett. 95, 133119-3 (2009)

7. W. Wang, P.N. Kumta. Acs Nano. 4, 2233 (2010)

8. S. Chen, M.L. Gordin, R. Yi, G. Howlett, H. Sohn, D. Wang. Phys. Chem. Chem. Phys. 14, 12741 (2012)
9. J. Shu, H. Li, R. Yang, Y. Shi, X. Huang. Electrochem. Commun. 8, 51-4 (2006)

10. K. Zhao, M. Pharr, L. Hartle, J.J. Vlassak, Z. Suo. J. Power Sources. 218, 6-14 (2012)

11. Z. Jia, W.K. Liu. J. Electrochem. Soc. 163, A940A6 (2016)

12. N. Ding, J. Xu, Y.X. Yao, G. Wegner, X. Fang, C.H. Chen. Solid State Ion. 180, 222-5 (2009)

13. K. Zhao, M. Pharr, J.J. Vlassak, Z. Suo. J. Appl. Phys. 109, 31 (2011)

14. M.N. Obrovac, L.J. Krause. J. Electrochem. Soc. 154, A103-A8 (2007)

15. V.B. Shenoy, P. Johari, Y. Qi. J. Power Sources. 195, 6825-30 (2010)

16. V.A. Sethuraman, M.J. Chon, M. Shimshak, V. Srinivasan, P.R. Guduru. J. Power Sources. 195, 5062-6 (2011)

17. H. Wang, S.P.V. Nadimpalli, V.B. Shenoy. Extreme Mech. Lett. (2016) 